\title{
Generating random correlation matrices with fixed values: An application to the evaluation of multivariate surrogate endpoints
}

\author{
ALVARO J. FLÓREZ* \\ I-BioStat, Universiteit Hasselt, B-3590 Diepenbeek, Belgium \\ alvaro.florez@uhasselt.be \\ ARIEL ALONSO ABAD \\ I-BioStat, KU Leuven, B-3000 Leuven, Belgium \\ GEERT MOLENBERGHS \\ I-BioStat, Universiteit Hasselt, B-3590 Diepenbeek, Belgium \\ I-BioStat, KU Leuven, B-3000 Leuven, Belgium \\ WIM VAN DER ELST \\ Janssen Pharmaceuticals, B-2340 Beerse, Belgium.
}

\begin{abstract}
SUMmaRY
When assessing surrogate endpoints in clinical studies under a causal-inference framework, a simulation-based sensitivity analysis is required, so as to sample the unidentifiable parameters across plausible values. To be precise, correlation matrices need to be sampled with only some of their entries identified from the data, known as the matrix completion problem. The positivedefiniteness constraints are cumbersome functions involving all matrix entries, making this a

*To whom correspondence should be addressed.
\end{abstract}


challenging task. Some existing algorithms rely on sampling and then rejecting invalid solutions. In this paper, we build on previous work and introduce a very efficient algorithm to generate correlation matrices with fixed elements. The proposed methodology is applied to tackle a difficult problem in the surrogate marker field, namely, the evaluation of multivariate, potentially highdimensional, surrogate endpoints. Whereas existing methods are limited to very low-dimensional surrogates, our proposal is stable, fast, shows good properties, and is implemented in a userfriendly and freely available R library.

Key words: Multiple surrogate evaluation; Partial correlation; Positive-definite matrix; Random correlation matrices; Simulation-based sensitivity analysis

\section{INTRODUCTION}

In the causal-inference framework, one frequently fits models with an only partially identifiable set of parameters $\boldsymbol{\theta}$, i.e., there is a subset of $\boldsymbol{\theta}$ that cannot be estimated from the data. A possible solution to this problem is to impose untestable restrictions, e.g., based on expert knowledge, for the unidentifiable parameters to estimate the model. Alternatively, one can conduct a sensitivity analysis to assess how the fitted model and conclusions based there upon change as the unidentifiable parameters vary across plausible values. The latter option is taken by Alonso and others (2015) and Van der Elst and others (2018) to evaluate univariate and multivariate surrogate endpoints in a causal-inference framework. Surrogacy is then evaluated using the so-called individual causal association (ICA), i.e., the association between the individual causal treatment effects on the surrogate and true endpoints. The ICA is a function of a partially identifiable correlation matrix $(\boldsymbol{R})$. Their approach rests upon computing the ICA across a set of randomly generated, valid (i.e., positive-definite) correlation matrices, but importantly, while keeping the estimable values fixed. This so-called matrix completion problem is non-trivial. 
A valid $(d \times d)$ correlation matrix $\boldsymbol{R}=\left(\rho_{i j}\right)$ not only requires a unit diagonal, and $-1 \leqslant$ $\rho_{i j}=\rho_{i j} \leqslant 1$, for $i \neq j$, but it also has to be positive-definite (PD). The latter constraint involves all the values of $\boldsymbol{R}$, and therefore, its random generation is very challenging. However, several algorithms have been presented. Joe (2006) proposes a method based on a transformation of partial correlations, later extended by Lewandowski and others (2009). Pourahmadi and Wang (2015) introduced an algorithm using the hyperspherical parametrization (HP) of the Cholesky factor. More alternatives can be found in Davies and Higham (2000); Mittelbach and others (2012); Madar (2015), among others.

To generate random correlation matrices with fixed values, some of the methods presented above need to be adapted. Joe's algorithm can be generalized by conveniently rearranging the fixed elements of the correlation matrix. On the other hand, the adaptation of the method proposed by Pourahmadi and Wang (2015) is cumbersome. Another alternative is to simulate a pseudo-correlation matrix and to find the nearest correlation matrix, according to some metric, keeping the identifiable values fixed. Some of these adjustments for non-positive-definiteness can be found in Rousseeuw and Molenberghs (1993) and Higham and others (2016).

In this paper, various algorithms to generate random correlation matrices with fixed values are introduced and evaluated. Furthermore, the methodology is applied to solve a difficult problem in the surrogate marker field, namely, the evaluation of multivariate surrogate endpoints, as well as in a different, high-dimensional context.

The structure of the manuscript is as follows. Section 2 presents the methodology for assessing multiple surrogates briefly. In Section 3, various algorithms to generate unrestricted random correlation matrices are introduced. Section 4 describes the algorithms to generate random correlation matrices with some of their values fixed. A simulation study to compare the methods is executed in Section 5. A motivating experiment on mice (The transPAT study) is presented and analyzed in Section 6. Section 7 is reserved for final remarks. 


\section{Assessing a multivariate surrogate}

In clinical trials, a true endpoint is defined as the most credible indicator of drug response. However, its measurement might be costly, difficult or requiring long follow-up time. Therefore, finding a less complex valid "substitute", termed a surrogate, of the true endpoint is very convenient (Burzykowski and others, 2005). In the last decades, several statistical methodologies to evaluate surrogate endpoints have been proposed, most of them within the causal-inference and meta-analytic paradigms. In this paper, we focus on the former. Details on surrogacy evaluation can be found in Burzykowski and others (2005), Molenberghs and others (2010), Alonso Abad and others (2016), among others.

We consider a single-trial setting: the data consist of measurements of a univariate true endpoint $T$ and a $p$-dimensional surrogate endpoint $\boldsymbol{S}=\left(S_{1}, \ldots, S_{p}\right)^{\prime}$ for $N$ patients. Moreover, only two treatments are under evaluation $(Z=0 / 1)$ in a parallel study design. Rubin's model for causal inference (Rubin, 1986) assumes that each patient has two potential outcomes for $T$ : an outcome $T_{0}$ that would be observed under the control treatment $(Z=0)$, and an outcome $T_{1}$ that would be observed under the experimental treatment $(Z=1)$. Furthermore, using evident notation, let us now consider the $2(p+1)$ dimensional vector of potential outcomes $\boldsymbol{Y}=\left(T_{0}, T_{1}, S_{10}, S_{11}, S_{20}, S_{21}, \ldots, S_{p 0}, S_{p 1}\right)^{\prime}$ and the corresponding vector of individual causal treatment effects $\boldsymbol{\Delta}=\left(\Delta T, \boldsymbol{\Delta} \boldsymbol{S}^{\prime}\right)^{\prime}$, where $\Delta T=T_{1}-T_{0}$ and $\boldsymbol{\Delta} \boldsymbol{S}=\left(\Delta S_{1}, \Delta S_{2}, \ldots \ldots, \Delta S_{p}\right)^{\prime}$ with $\Delta S_{k}=S_{k 1}-S_{k 0}$. The so-called fundamental problem of causal inference states that only one of the potential outcomes associated with the true and surrogate endpoints are observed in practice. Therefore, $\boldsymbol{\Delta}$ cannot be estimated from the data (Holland, 1986). Note that, to avoid clutter, no subindex has been used to denote the patient.

Based on $\boldsymbol{\Delta}$, one can define the expected or average causal treatment effects in the population of interest as $E(\boldsymbol{\Delta})=(\beta, \boldsymbol{\alpha})$, where $\beta=E(\Delta T)$ and $\boldsymbol{\alpha}=\left(\alpha_{k}\right)$ with $\alpha_{k}=E\left(\Delta S_{k}\right)$.

Rosenbaum and Rubin (1983) provided three identifiability conditions under which it is pos- 
sible to obtain consistent estimators of the expected causal treatment effects. If $Y$ denotes the response of interest and $Y_{z}$ the potential outcome associated with $Z=z$ then the three identifiability conditions are: 1) Consistency: If $Z=z$ for a given subject then $Y_{z}=Y$ for that subject, 2) Conditional exchangeability: This condition essentially states that there are not unmeasured confounding given data on baseline covariates $L$, that is, $Y_{z} \perp Z \mid L=l$ for each possible value $z$ of $Z$ and $l$ of $L$ and 3) Positivity: If $f_{L}(l) \neq 0$ then $f_{Z \mid L}(z \mid l)>0$. It can easily be shown that in randomized clinical trials all condition hold and the expected causal treatment effects can be estimated as $\beta=E(T \mid Z=1)-E(T \mid Z=0)$ and $\alpha_{k}=E\left(S_{k} \mid Z=1\right)-E\left(S_{k} \mid Z=0\right)$, where the conditional expectations are estimated using the observed means in the control and treated groups, respectively. The metric of surrogacy proposed by Van der Elst and others (2018), and used in the following sections, is based only on the individual causal treatment effects and it is valid if consistency holds, i.e., it could also be applied to observational data.

In the surrogacy evaluation context, one is interested in the distribution of the vector of potential outcomes $\boldsymbol{Y}$. Consequently, it will be further assumed that $\boldsymbol{Y} \sim N(\boldsymbol{\mu}, \boldsymbol{\Sigma})$, where $\boldsymbol{\mu}=$ $\left(\mu_{T_{0}}, \mu_{T_{1}}, \mu_{S_{10}}, \mu_{S_{11}}, \ldots, \mu_{S_{p 0}}, \mu_{S_{p 1}}\right)^{\prime}$ and

$$
\boldsymbol{\Sigma}=\left(\begin{array}{ccccccccc}
\sigma_{T_{0} T_{0}} & \sigma_{T_{0} T_{1}} & \sigma_{T_{0} S_{10}} & \sigma_{T_{0} S_{11}} & \sigma_{T_{0} S_{20}} & \sigma_{T_{0} S_{21}} & \ldots & \sigma_{T_{0} S_{p 0}} & \sigma_{T_{0} S_{p 1}} \\
& \sigma_{T_{1} T_{1}} & \sigma_{T_{1} S_{10}} & \sigma_{T_{1} S_{11}} & \sigma_{T_{1} S_{20}} & \sigma_{T_{1} S_{21}} & \ldots & \sigma_{T_{1} S_{p 0}} & \sigma_{T_{1} S_{p 1}} \\
& & \sigma_{S_{10} S_{10}} & \sigma_{S_{01} S_{20}} & \sigma_{S_{01} S_{11}} & \sigma_{S_{01} S_{21}} & \ldots & \sigma_{S_{01} S_{p 0}} & \sigma_{S_{01} S_{p 1}} \\
& & & \sigma_{S_{11} S_{11}} & \sigma_{S_{11} S_{20}} & \sigma_{S_{11} S_{21}} & \ldots & \sigma_{S_{11} S_{p 0}} & \sigma_{S_{11} S_{p 1}} \\
& & & & \sigma_{S_{20} S_{20}} & \sigma_{S_{20} S_{21}} & \ldots & \sigma_{S_{20} S_{p 0}} & \sigma_{S_{20} S_{p 1}} \\
& & & & \sigma_{S_{21} S_{21}} & \ldots & \sigma_{S_{21} S_{p 0}} & \sigma_{S_{21} S_{p 1}} \\
& & & & & \ddots & \vdots & \vdots \\
& & & & & & \sigma_{S_{p 0} S_{p 0}} & \sigma_{S_{p 0} S_{p 1}} \\
& & & & & & & \sigma_{S_{p 1} S_{p 1}}
\end{array}\right) .
$$

Under the previous assumptions, one has that $\boldsymbol{\Delta} \sim N\left(\boldsymbol{\mu}_{\boldsymbol{\Delta}}, \boldsymbol{\Sigma}_{\boldsymbol{\Delta}}\right)$, with $\boldsymbol{\mu}_{\boldsymbol{\Delta}}=(\beta, \boldsymbol{\alpha})^{\prime}$ and

$$
\boldsymbol{\Sigma}_{\boldsymbol{\Delta}}=\left(\begin{array}{cc}
\sigma_{\Delta T} & \boldsymbol{\Sigma}_{\boldsymbol{\Delta} \boldsymbol{S} \Delta T}^{\prime} \\
\boldsymbol{\Sigma}_{\boldsymbol{\Delta} \boldsymbol{S} \Delta T} & \boldsymbol{\Sigma}_{\boldsymbol{\Delta} \boldsymbol{S}}
\end{array}\right),
$$

where $\sigma_{\Delta T}=\sigma_{T_{0} T_{0}}+\sigma_{T_{1} T_{1}}-2 \rho_{T_{0} T_{1}} \sqrt{\sigma_{T_{0} T_{0}}+\sigma_{T_{1} T_{1}}}$ is the variance of $\Delta T ; \boldsymbol{\Sigma}_{\Delta \boldsymbol{S} \Delta T}$ is a $p$ dimensional vector of covariances between $\Delta T$ and $\boldsymbol{\Delta} \boldsymbol{S}=\left(\Delta S_{1}, \Delta S_{2}, \ldots, \Delta S_{p}\right)^{\prime} ;$ and $\boldsymbol{\Sigma}_{\boldsymbol{\Delta} \boldsymbol{S}}$ is the $(p \times p)$ variance-covariance matrix of $\boldsymbol{\Delta} \boldsymbol{S}$. 


\subsection{Individual causal association based on a multivariate surrogate}

In the univariate setting $(p=1)$, Alonso and others (2015) defined the ICA as the Pearson correlation coefficient between $\Delta T$ and $\Delta S$ :

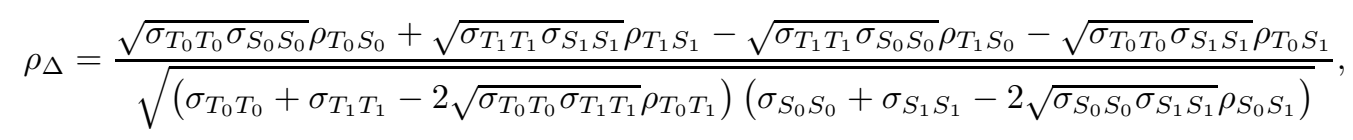

where $\rho_{X Y}$ is the correlation between the potential outcomes $X$ and $Y$. It quantifies how accurate the prediction is of the causal treatment effect on the true endpoint using the causal treatment effect on the surrogate, for a given patient.

Only the variances and correlations $\rho_{T_{0} S_{0}}, \rho_{T_{1} S_{1}}$ are identifiable from the data. Therefore, the ICA cannot be identified without imposing untestable restricitons on the unidentifiable correlations. Hence, Alonso and others (2015) proposed a simulation-based sensitivity analysis in which $\rho_{\Delta}$ is calculated across a set of plausible values for the inestimable elements in (2.2). To be precise, they considered all positive-definite matrices over a grid of values $G=\left(g_{1}, \ldots, g_{k}\right)$, with $-1 \leqslant g_{i} \leqslant 1$ for the unidentifyable correlations, and then calculated $\rho_{\Delta}$ for each of them. The so-obtained collection produces an insightful distribution that can be examined graphically, or summarized using appropriate point and/or interval summaries.

For the case of a multivariate surrogate, Van der Elst and others (2018) proposed the so-called squared information coefficient of correlation (SICC; Linfoot, 1957; Joe, 1989) to quantify the ICA, i.e.,

$$
R_{H}^{2}=\frac{\boldsymbol{\Sigma}_{\Delta \boldsymbol{S} \Delta T}^{\prime} \boldsymbol{\Sigma}_{\boldsymbol{\Delta} \boldsymbol{S}}^{-1} \boldsymbol{\Sigma}_{\boldsymbol{\Delta} \boldsymbol{S} \Delta T}}{\sigma_{\Delta T}}
$$

The $R_{H}^{2}$ range over the unit interval $[0,1]$, and takes the value zero if and only $\Delta T$ and $\Delta \boldsymbol{S}$ are independent, while it takes the value one if and only if $\Delta T$ is perfectly linearly predictable from $\boldsymbol{\Delta} \boldsymbol{S}$. As in the univariate case, $R_{H}^{2}$ is not identifiable from the data, and a simulation-based sensitivity analysis is recommended. However, the grid-based approach becomes computationally too intensive, even infeasible, as the number of surrogate endpoints increases. While of course 
there is the effect of increasing dimensions, the main issue is that with increasing dimensions, the space of positive-definite matrices is an ever smaller subset of the rectangle $[-1,+1]^{d}$ with $d$ the number of functionally different correlations involved. Therefore, this approach, rejection sampling, to be discussed in the next section, is limited to a small number of surrogates.

\section{Generating RANDOM CORRELATiOn MATRICES}

\subsection{Rejection sampling $(R S)$ algorithm}

In this method, $\boldsymbol{R}$ is constructed by drawing $\rho_{i j}$ independently from a distribution on $(-1,1)$, e.g., uniform. If the generated $\tilde{\boldsymbol{R}}$ matrix is not PD, it is rejected. This method performs well when the order of the correlation matrix is small. However, the rejection rate increases rapidly with $d$, making it time-consuming or even not possible. Simulation studies show that the RS algorithm generates almost no valid correlation matrices for $d>7$ (Numpacharoen and Atsawarungruangkit, 2012; Böhm and Hornik, 2014).

To decrease the rejection rate and computing time, the RS algorithm can be implemented in a gradual way using Sylvester's criterion, i.e., a matrix is PD when all the upper-left sub-matrices have positive determinants. Thus, to generate a $(d \times d)$ correlation matrix, we start randomly sampling the upper-left $(2 \times 2)$ sub-matrix using the RS algorithm. When the determinant is positive, the same procedure is used for the upper-left $(3 \times 3)$ sub-matrix, and so on.

\section{$3.2 \quad$ Algorithm based on partial correlations (PC)}

Joe (2006) proposed to generate a random correlation matrix $\boldsymbol{R}$ progressively based on a parameterization in terms of the correlations $\rho_{i, i+1}$ for $i=1, \ldots, d-1$ and the partial correlations $\rho_{i j \mid i+1, \ldots, j-1}$ for $j-i \geqslant 2$. The algorithm is based on the following equality:

$$
\rho_{j, j+k}=\boldsymbol{r}_{1}^{\prime}(j, k)\left\{\boldsymbol{R}_{2}(j, k)\right\}^{-1} \boldsymbol{r}_{3}(j, k)+\rho_{j, j+k \mid j+1, \ldots, j+k-1} D_{j, k},
$$


where

$$
\boldsymbol{R}[j: j+k]=\left(\begin{array}{ccc}
1 & \boldsymbol{r}_{1}^{\prime}(j, k) & \rho_{j, j+k} \\
\boldsymbol{r}_{1}(j, k) & \boldsymbol{R}_{2}(j, k) & \boldsymbol{r}_{3}(j, k) \\
\rho_{j+k, j} & \boldsymbol{r}_{3}^{\prime}(j, k) & 1
\end{array}\right)
$$

with $\boldsymbol{r}_{1}^{\prime}(j, k)=\left(\rho_{j, j+1}, \ldots, \rho_{j, j+k-1}\right), \boldsymbol{r}_{3}^{\prime}(j, k)=\left(\rho_{j+k, j+1}, \ldots, \rho_{j+k, j+k-1}\right), \boldsymbol{R}_{2}(j, k)$ being the middle $(k-1 \times k-1)$ matrix of $\boldsymbol{R}[j: j+k]$, and:

$$
D_{j, k}=\sqrt{\left\{1-\boldsymbol{r}_{1}^{\prime}(j, k) \boldsymbol{R}_{2}(j, k) \boldsymbol{r}_{1}(j, k)\right\}\left\{1-\boldsymbol{r}_{3}^{\prime}(j, k) \boldsymbol{R}_{2}(j, k) \boldsymbol{r}_{3}(j, k)\right\}} .
$$

Then, one can generate $\rho_{j, j+k \mid j+1, \ldots, j+k-1}(1 \leqslant k \leqslant d-1)$ independently in the interval $(-1,1)$ and then use $(3.4)$ to get $\rho_{i, i+k}$ for $2 \leqslant k \leqslant d-1$. Furthermore, the correlations $\rho_{j, j+1}$ are also independently generated in the interval $(-1,1)$.

To obtain identical symmetric marginal densities of each $\rho_{i, j}$, Joe (2006) proposes to draw each $\rho_{j, j+1}(j=1, \ldots, d-1)$ from a $\operatorname{Beta}\left(\frac{d}{2}, \frac{d}{2}\right)$ on $(-1,1)$, and each $\rho_{j, j+k \mid j+1, \ldots, j+k-1}$ from a $\operatorname{Beta}\left\{1+\frac{1}{2}(d-1-k), 1+\frac{1}{2}(d-1-k)\right\}$ on $(-1,1)$.

\subsection{Algorithm based on the hyperspherical parameterization of the Cholesky factor (HP)}

Pourahmadi and Wang (2015) introduced a method based on the reparameterization of the Cholesky factor of $\boldsymbol{R}$ using hyperspherical coordinates. Following the Cholesky factorization, a PD correlation matrix $\boldsymbol{R}$ can be factorized by $\boldsymbol{R}=\boldsymbol{U} \boldsymbol{U}^{\prime}$, where $\boldsymbol{U}=\left(u_{i j}\right)$ is a lower triangular matrix with $u_{11}=1, u_{i 1}=\cos \theta_{i, 1}$, for $i=1, \ldots, d$, and

$$
u_{i j}= \begin{cases}\prod_{k=1}^{j-1} \sin \theta_{i k} & \text { for } i=j \\ \cos \theta_{i j} \prod_{k=1}^{j-1} \sin \theta_{i k} & \text { for } 2 \leqslant j \leqslant i-1\end{cases}
$$

where $\theta_{i j}, i>j$, are angles restricted to $(0, \pi)$. Furthermore, the transformation from $\boldsymbol{R}$ to $\boldsymbol{\Theta}=\left(\theta_{i j}\right)$ is one-to-one.

Then, a random correlation matrix $\boldsymbol{R}$ is generated by drawing values of the $j$-th columns of the lower-triangular matrix $\boldsymbol{\Theta}$ using the following distribution:

$$
\theta_{i j} \sim g_{j}(\theta) \propto(\sin \theta)^{2 k+n-j} I(0<\theta<\pi), i=j+1, \ldots, d,
$$


where $k \geqslant 0$; and later, computing the lower triangular matrix $\boldsymbol{U}$ using $\theta_{i, j}$ and constructing the correlation matrix $\boldsymbol{R}=\boldsymbol{U} \boldsymbol{U}^{\prime}$.

For $k=0$, each $\rho_{i j}$ follows a $\operatorname{Beta}\left(\frac{d}{2}, \frac{d}{2}\right)$ distribution on $(-1,1)$, leading to the same distribution as the algorithm proposed by Joe (2006).

\section{Generating correlation matrices with fixed values}

With some correlations fixed, the same algorithms presented in Section 3 can be implemented. The RS and gradual-RS algorithms can be applied directly by randomly drawing the non-fixed $\rho_{i, j}$ values independently from a uniform distribution on $(-1,1)$ and rejecting the matrix if it is not PD. The latter is implemented by Van der Elst and others (2018) to compute $R_{H}^{2}$. However, they concluded that this algorithm is computationally suitable to consider at most four surrogates, which corresponds to generate random correlations matrices of size 10. For a larger number of surrogates, it breaks down.

The PC algorithm can be implemented by conveniently rearranging the correlation matrix and gradually sampling the unidentifiable $\rho_{i, j}$ values using (3.4). On the other hand, the adaptation of the HP algorithm is not straightforward. Translating the constraints on $\rho_{i, j}$ to the angles $\theta_{i, j}$ is cumbersome and unpractical, even for small $d$.

Another alternative is to generate a random correlation matrix $\left(\boldsymbol{R}^{(0)}\right)$ using the HP algorithm, replacing the prior fixed values to yield $\boldsymbol{R}^{(1)}$. If the $\boldsymbol{R}^{(1)}$ is not PD, we can find the nearest PD correlation matrix using the weighted scaling or linear shrinking method (Rousseeuw and Molenberghs, 1993). 


\subsection{Weighted scaling method}

Here, we find the PD correlation matrix $\tilde{\boldsymbol{R}}=\left(\tilde{r}_{i, j}\right)$ that is as near as possible to the pseudocorrelation matrix $\boldsymbol{R}^{(1)}=\left(r_{i, j}^{(1)}\right)$ using the following criterion:

$$
S=\sum_{i=1}^{d} \sum_{i=1}^{d} w_{i j}\left(r_{i j}^{(1)}-\tilde{r}_{i j}\right)^{2}
$$

where $w_{i j}=w_{j i}$ is the weight associated to correlation $r_{i j}$. Given that the PD constraint on $\tilde{\boldsymbol{R}}$ involves all $\tilde{r}_{i j}$ simultaneously, the minimization of (4.6) seems intractable. However, this problem is overcome by describing $\tilde{\boldsymbol{R}}$ in a geometric way (Section 3.3), and then, iteratively finding the angles $\theta_{i j}$ that minimize (4.6). By minimizing $S$ as a function of $\theta_{i j}$, the PD constraint on $\tilde{\boldsymbol{R}}$ is incorporated. Since $S$ is differentiable with respect to $\theta_{i j}$, we can apply iterative numerical algorithms, e.g., the steepest descent method. As initial values, we can use the $\theta_{i j}$ associated to $\boldsymbol{R}^{(1)}$.

To ensure that the fixed values do not move, or do so only very slightly, we set the corresponding weights to a very large value (e.g., $10^{5}$ ) and, the weights for elements that are allowed to change are set equal to a small value (one for example).

\subsection{Linear shrinking method}

Here, the pseudo-correlation matrix $\boldsymbol{R}^{(1)}$ is shrunk towards an arbitrary correlation matrix $\boldsymbol{R}^{(0)}$ according to,

$$
\tilde{\boldsymbol{R}}=\lambda \boldsymbol{R}^{(1)}+(1-\lambda) \boldsymbol{R}^{(0)},
$$

where $\lambda$ is the largest value in $[0,1]$ which makes $\tilde{\boldsymbol{R}}$ PD. In our case, $\boldsymbol{R}^{(0)}$ contains zero values for all correlations, except for the fixed ones. Then, to find $\tilde{\boldsymbol{R}}^{(1)}$, we proceed as follows: (1) find $\lambda$ in (4.7), (2) generate a random value $\tilde{\lambda}$ in $(0, \lambda)$, using an uniform distribution, for example, and (3) compute $\tilde{\boldsymbol{R}}$ according to (4.7) using $\tilde{\lambda}$. 


\section{Simulation Study}

To assess the performance of the previously introduced algorithms, a simulation study was carried out. The main objective of the simulation was to evaluate the computational feasibility of the different algorithms, i.e., to assess the time required to draw a random positive definite matrix. In addition, the generated random matrices were used to evaluate the validity of a putative multivariate surrogate endpoint $\boldsymbol{S}=\left(S_{1}, \ldots, S_{p}\right)^{\prime}$ for a univariate true endpoint $(T)$ using the ICA.

\section{$5.1 \quad$ Settings}

The identifiable correlations will be assumed equal, while different values will be considered: (a) for the number of surrogates, and therefore the matrix size $(d),(\mathrm{b})$ and for the values of the fixed identifiable correlations:

- Number of surrogates $(p)$ : one surrogate ( $4 \times 4$ matrix - two fixed values), three surrogates $(8 \times 8$ matrix -12 fixed values $)$, five surrogates $(12 \times 12$ matrix -30 fixed values $)$ and 10 surrogates $(22 \times 22$ matrix -110 fixed values $)$.

- Level of correlation $(\rho)$ : low $(\rho=0.2)$, moderate $(\rho=0.5)$, and high $(\rho=0.8)$ correlation.

For conciseness, the identifiable correlations are fixed at the same value in the simulation settings. However, none of the algorithms require this. We performed additional simulations with correlation matrices in which the identifiable elements were not all equal. Results turn out to be very similar. One of these simulations is presented in Section A.2 in supplementary materials available at Biostatistics online.

For each scenario, we implemented four different methodologies: (1) the gradual rejection sampling algorithm (GRS), (2) the algorithm based on partial correlation (HP), (3) the shrinking method (SHR), and (4) the scaling method (SCA). A total of 1,000 random correlation matrices 
were generated with each method. As outcome, we are interested in the computation time to draw a correlation matrix with fixed values. Furthermore, we computed the univariate $\left(\rho_{\Delta}\right.$; for $p=1)$ and multivariate $\left(R_{H}^{2}\right.$; for $\left.p>1\right)$ ICA using the generated random correlation matrices. Here, we are interested in comparing the densities of the resulting ICA quantities.

\subsection{Results}

Table 1 shows the expected time to generate a random correlation matrix with fixed values using the four methods. The GRS algorithm works well for matrices of sufficiently small dimension, around eight say, when the magnitude of the fixed correlations is sufficiently small. At the same time, it is almost impossible to find a valid correlation matrix when the dimension is 12 or larger and/or when the fixed correlations are relatively large in absolute value. To draw a $(12 \times 12)$ matrix and $\rho=0.8$, it takes more than five minutes, making it impractical. Both methods based on adjustments for non-positive-definiteness are fast, with a longer time for the SCA method. However, they do not perform well with high-dimensional matrices. The SCA method fails to keep fixed the identifiable correlations for matrices of size greater than four, even using very large weights for the fixed values. On the other hand, the shrinking parameter $(\alpha)$ goes to zero as the matrix size increases, leading to draws closer to 0 for the SHR method (for more details, see Section A.1 in supplementary materials available at Biostatistics online). Our algorithm based on partial correlations is the fastest and generates correlations with constant symmetric densities (see Figure A.1 in supplementary materials available at Biostatistics online).

The frequency densities for the ICA obtained from the different methods are displayed in Figure 1. The GRS and HP algorithm provide similar frequency densities for the ICA in all scenarios where the latter are feasible. The SHR method leads to a more narrow and more peaked distribution of the ICA when $d$ and $\rho$ increase. The SCA algorithm provides results similar to those of the GRS and HP methods when the fixed correlations are low. However, it does not 
behaves well for $\rho=0.5$ or $\rho=0.8$. Given that it fails to keep fixed the identifiable correlations, it leads to invalid values for the ICA. Note that we are using the GRS method as the reference when analyzing the ICA densities.

\subsection{Identifiable bounds: Additional simulation}

One of the advantages of the simulation-based sensitivity analysis introduced by Alonso and others (2015) and Van der Elst and others (2018), is that it can provide approximate identifiable bounds for the unidentifiable ICA. An additional simulation study was conducted to evaluate the performance of these identifiable bounds. The bounds are calculated based on $M$ runs of the PC algorithm. Indeed, if the number of simulated correlation matrices is sufficiently large and the PC algorithm samples from the entire space of correlation matrices, producing the corresponding

$R_{H}^{2}$ values, then the observed ( $\min R_{H}^{2}$, $\max R_{H}^{2}$ ) should contain the true value of the ICA and, therefore, they can be considered as approximate bounds for the true ICA.

For the simulations, a setting with a bivariate $\boldsymbol{S}=\left(S_{1}, S_{2}\right)$ surrogate endpoint was considered. Three positive definite matrices were generated using the GRS algorithm. These matrices led to ICA values equal to $0.75,0.85$ and 0.95 . Based on each of these matrices, 250 datasets of sizes $N=\{100,200,500\}$ were generated. When analyzing each dataset, the unidentifiable quantities were removed and the bounds for the ICA were computed by $M=\{100 ; \ldots ; 50,000\}$ runs of the PC algorithm. Table 2 shows the percentage of cases in which the true ICA was included in the range of the calculated $R_{H}^{2}$ (coverage).

When $N=500$ and a minimum of $M=1,000$ runs are used, valid bounds are generally obtained, i.e, the coverage probability exceeds $90 \%$ in all cases and it is often larger than 95\%. However, when the ICA equals 0.75 and the sample size is relatively small more runs may be necessary to obtained satisfactory bounds. Extra simulations showed that the GRS algorithm provides reasonably similar coverage rates as the ones exhibited in Table 2, at least with 
$M=\{100,200,500\}$. The coverage evaluation for higher numbers of runs of this algorithm is computationally too time-consuming.

\section{The transPAT MiCROBIOME INTERVENTION STUDY}

The TransPAT experiment (Ruiz and others, 2017) is an animal study conducted to evaluate the influence of an antibiotic treatment on the immune system (Immunoglobulin A level, IgA level). The dataset consists of information from 15 germ-free mice that received cecal contents of a donor mouse. The cecal contents of seven donor mice were exposed to a tylosin pulse (experimental treatment group) and eight mice were not exposed (control treatment group). 12 days after starting the experiment, the relative abundance of a total of 67 operational taxonomic units (OTUs) was measured. Regarding multiple surrogates assessment, the objective of the study was to evaluate whether the treatment effect on one or more OTUs (candidate surrogate endpoints) conveys information on the potential treatment effect on the immune response (IgA level at day 20 ; true endpoint). The data are available on github (see https://github.com/blaser-lab/ Paper-Ruiz-2017) and was used by Van der Elst and others (2018) to illustrate the multiple surrogates evaluation methodology.

\subsection{Assessing the validity of a multivariate surrogate in the case study}

Van der Elst and others (2018) conducted a simulation-based sensitivity analysis where the $R_{H}^{2}$ was computed using random correlation matrices simulated by the gradual rejection sampling algorithm (GRS). Since the number of potential surrogate endpoints is large $(p=67)$, they opted for a forward selection approach to identify the best set of surrogates. At first, a univariate analysis for each candidate surrogate is performed. Then, the one with the highest median $R_{H}^{2}$ is kept. Afterwards, multivariate analyses are conducted, including the first chosen surrogate combined with each of the remaining candidates. Then, the pair of surrogates with the highest median 
$R_{H}^{2}$ is retained. The procedure continues until the group of selected surrogates reach a criterion, e.g., until the median $R_{H}^{2}>0.95$. In this Section, we proceed in the same way but using the PC algorithm generating 10,000 random correlation matrices. Section A.3 in the supplementary materials available at Biostatistics online shows how to conduct a multiple surrogacy analysis using the Surrogate R package.

Figure 2 (left hand side) shows the median $R_{H}^{2}$ of all sets of surrogates evaluated in each step. In the univariate evaluation, not all the candidates seem to be good, several medians $R_{H}^{2}$ are very small. Nevertheless, the median of the best one $\left(S_{1}\right)$ is relatively high. As more candidates are jointly evaluated, the medians increase. In the third step, all the evaluations show a median higher than 0.85 . Figure 2 (right hand side) displays the range of $R_{H}^{2}$ values obtained from the best combination of surrogates in each step. When the best candidate surrogate is individually evaluated, the $R_{H}^{2}$ exhibits a wide range, indicating a strong impact of the unverifiable parameters. However, the range gets narrower as more surrogates are simultaneously assessed. With five surrogates, the median $R_{H}^{2}$ reaches 0.976 with a minimum of 0.922 and a maximum of 0.999 . Until this step, the selected vector of surrogates corresponds to $S_{1}=$ OTU $44, S_{2}=$ OTU 17 , $S_{3}=$ OTU $37, S_{3}=$ OTU 40 and $S_{3}=$ OTU $30\left(S_{1}\right.$ belongs to the family Ruminococcaceae, $S_{2}$ to Verrucomicrobiaceae, and $S_{3}, S_{4}$ and $S_{5}$ are members of the Lachnospiraceae family).

Van der Elst and others (2018) performed the forward selection approach until step three, finding fairly the same results for the $R_{H}^{2}$. However, they selected a different set of surrogates after the first step. This can be explained by the fact that the bivariate analysis of most of the other candidates and $S_{1}$ provide high $R_{H}^{2}$ values (see Figure 2, left hand side). Around $15 \%$ of the them combined with $S_{1}$ lead to a median $R_{H}^{2}$ higher than $85 \%$.

The median time to generate 500 correlation matrices in the evaluation of one to all surrogates is presented in Figure 3. The evaluation of a small number of surrogates is fast, e.g., around 3 seconds to assess five surrogates, but the computation time increases exponentially. Nevertheless, 
the PC algorithm can still generate large random correlation matrices in a relatively short time. The evaluation of 67 surrogates, i.e., simulating $(136 \times 136)$ correlation matrices with 4,488 fixed values, took around 56 minutes.

\section{FinAL REMARKS}

Different methods of generating random correlation matrices were adapted to cases where some of the single correlations are fixed, a typical situation in the causal-inference framework. The simulation study showed that all methods show reasonably similar performance with small size matrices. However, for medium to high dimensions, the algorithm based on partial correlations (PC) outperforms the others. It is fast and is not affected by the magnitude of the identifiable correlations.

In the multiple surrogates assessment, the computation of the $R_{H}^{2}$ by the GSR method is computationally intensive even with few surrogates, and unfeasible with more than five, limiting the joint evaluation to a relatively small number of candidates. On the other hand, the PC algorithm allows assessing more surrogates in a short time. Nevertheless, in practical cases is not necessary to evaluate a large number of surrogates combined. However, the PC algorithm is not only limited to this framework. It can be used with high-dimensional matrices. In the case study, we were able to simulate random correlation matrices of size $(136 \times 136)$ (evaluating all candidates combined) in a relatively fast manner. Furthermore, it allows incorporating additional restrictions about the unidentifiable correlations easily, e.g., biologically implausible values. However, this may affect the marginal distribution of the single correlations considerably.

\section{SupPlementary Materials}

The reader is referred to the online Supplementary Materials available at http://biostatistics . oxfordjournals.org for additional simulations, and an illustration of the R package Surrogate. 


\section{ACKNOWLEDGMents}

Financial support from the IAP research network \#P7/06 of the Belgian Government (Belgian Science Policy) is gratefully acknowledged. Alvaro J. Flórez acknowledges funding from the European Seventh Framework programme FP7 2007 - 2013 under grant agreement Nr. 602552.

\section{REFERENCES}

Alonso, Ariel, der Elst, Wim Van, Molenberghs, Geert, Buyse, Marc and Burzykowski, Tomasz. (2015). On the relationship between the causal-inference and metaanalytic paradigms for the validation of surrogate endpoints. Biometrics $\mathbf{7 1}(1), 15-24$.

Alonso Abad, A., Bigirumurame, T., Burzykowski, T., Buyse, M., Molenberghs, G., Muchene, L., Perualila, N.J., Shkedy, Z. and Van der Elst, W. (2016). Applied Surrogate Endpoint Evaluation with SAS and R. Boca Ratón: Chapman \& Hall/CRC.

BöHm, W. AND HoRNiK, K. (2014). Generating random correlation matrices by the simple rejection method: Why it does not work. Statistics \& Probability Letters 87, 27-30.

Burzykowski, T., Molenberghs, G. And Buyse, M. (2005). The Evaluation of Surrogate Endpoints. Springer-Verlag GMBH.

Davies, P. I. AND Higham, N. J. (2000). Numerically stable generation of correlation matrices and their factors. BIT Numerical Mathematics 40(4), 640-651.

Higham, Nicholas, Strabić, Nataa and Šego, Vedran. (2016). Restoring definiteness via shrinking, with an application to correlation matrices with a fixed block. SIAM Review 58(2), $245-263$.

Holland, P. W. (1986). Statistics and causal inference. Journal of the American Statistical Association 81(396), 945-960. 
Joe, H. (1989). Relative entropy measures of multivariate dependence. Journal of the American Statistical Association 84(405), 157-164.

JoE, H. (2006). Generating random correlation matrices based on partial correlations. Journal of Multivariate Analysis 97(10), 2177-189.

Lewandowski, D., Kurowicka, D. and Joe, H. (2009). Generating random correlation matrices based on vines and extended onion method. Journal of Multivariate Analysis 100(9), $1989-2001$.

Linfoot, E.H. (1957). An informational measure of correlation. Information and Control 1(1), $85-89$.

MADAR, VERED. (2015). Direct formulation to cholesky decomposition of a general nonsingular correlation matrix. Statistics \& Probability Letters 103, 142-147.

Mittelbach, Martin, Matthiesen, Bho and Jorswieck, Eduard A. (2012). Sampling uniformly from the set of positive definite matrices with trace constraint. IEEE Transactions on Signal Processing 60(5), 2167-2179.

Molenberghs, G., Burzykowski, T., Alonso, A., Assam, P., Tilahun, A. and Buyse, M. (2010). A unified framework for the evaluation of surrogate endpoints in mental-health clinical trials. Statistical Methods in Medical Research 19(3), 205-236.

Numpacharoen, K. And AtsaWarungruangkit, A. (2012). Generating correlation matrices based on the boundaries of their coefficients. PLoS ONE 7(11), 1-7.

Pourahmadi, Mohsen And Wang, XiaO. (2015). Distribution of random correlation matrices: Hyperspherical parameterization of the cholesky factor. Statistics $\&$ Probability Letters 106, $5-12$. 
Rosenbaum, P. R. and Rubin, D. B. (1983). The central role of the propensity score in observational studies for causal effects. Biometrika $\mathbf{7 0}(1), 41-55$.

Rousseeuw, P. J. And Molenberghs, G. (1993). Transformation of non positive semidefinite correlation matrices. Communications in Statistics, Theory and Methods 22(4), 965-984.

Rubin, D. B. (1986). Statistics and causal inference: Comment: which ifs have causal answers. Journal of the American Statistical Association 81(396), 961-962.

Ruiz, Victoria E., Battaglia, Thomas, Kurtz, Zachary D., Bijnens, Luc, Ou, Amy, Engstrand, Isak, Zheng, Xuhui, Iizumi, Tadasu, Mullins, Briana J., Mller, Christian L., Cadwell, Ken, Bonneau, Richard, Perez-Perez, Guillermo I. and others. (2017). A single early-in-life macrolide course has lasting effects on murine microbial network topology and immunity. Nature Communications 8(1).

Van der Elst, W., Alonso, A., Geys, H., Meyvisch, P., Bijnens, L. And Molenberghs, G. (2018). Univariate versus multivariate surrogate endpoints. [submitted].

[Received August 1, 2010; revised October 1, 2010; accepted for publication November 1, 2010] 

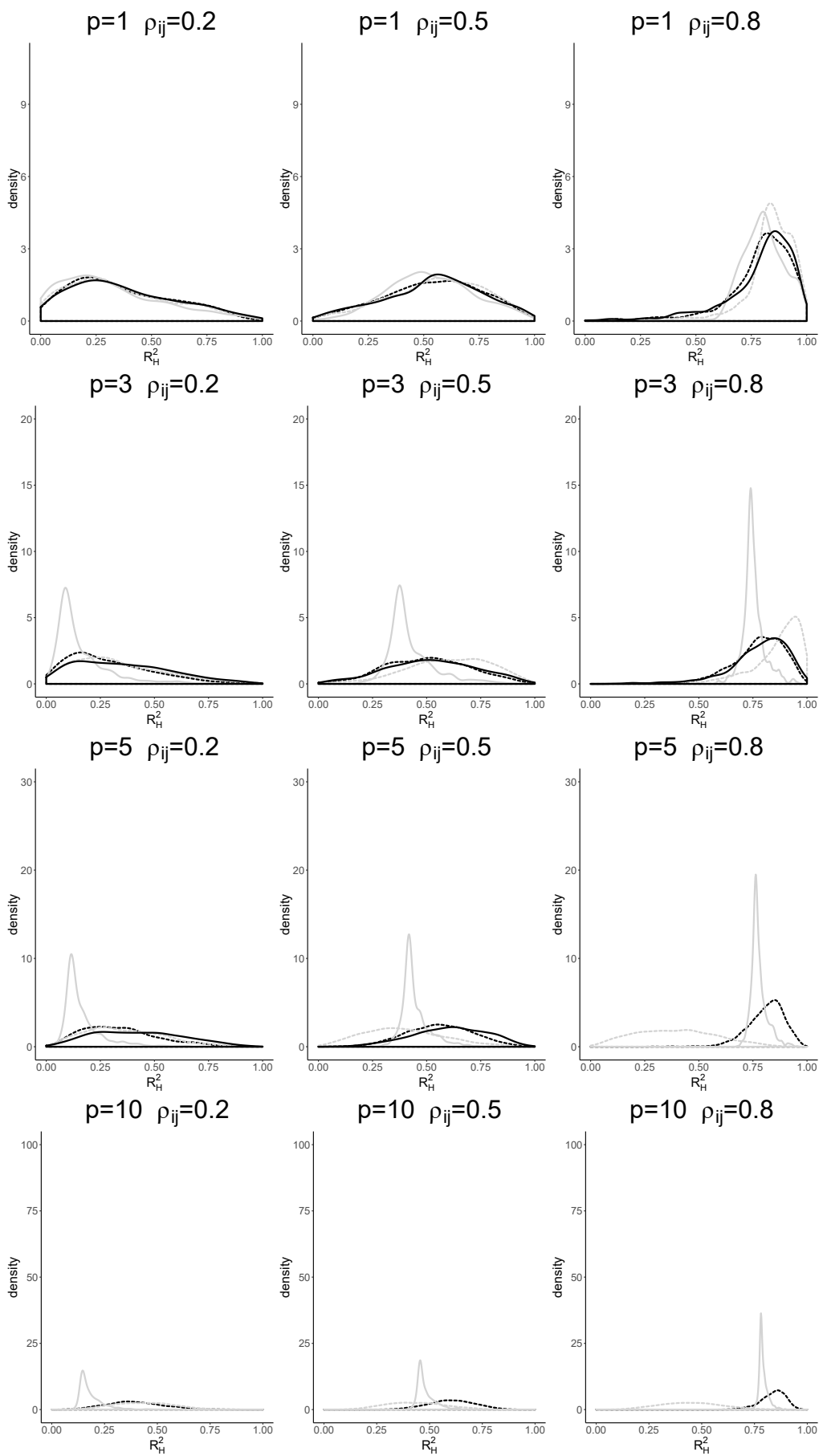

Fig. 1. Density of the univariate and multivariate ICA computed using four methods to generate the unidentifiable correlations: gradual rejection sampling (solid black lines), algorithm based on partial correlations (dashed black line), linear shrinking method (solid grey line) and scaling method (dashed grey line) for different number of surrogates $(p)$ and fixed $\rho$. 

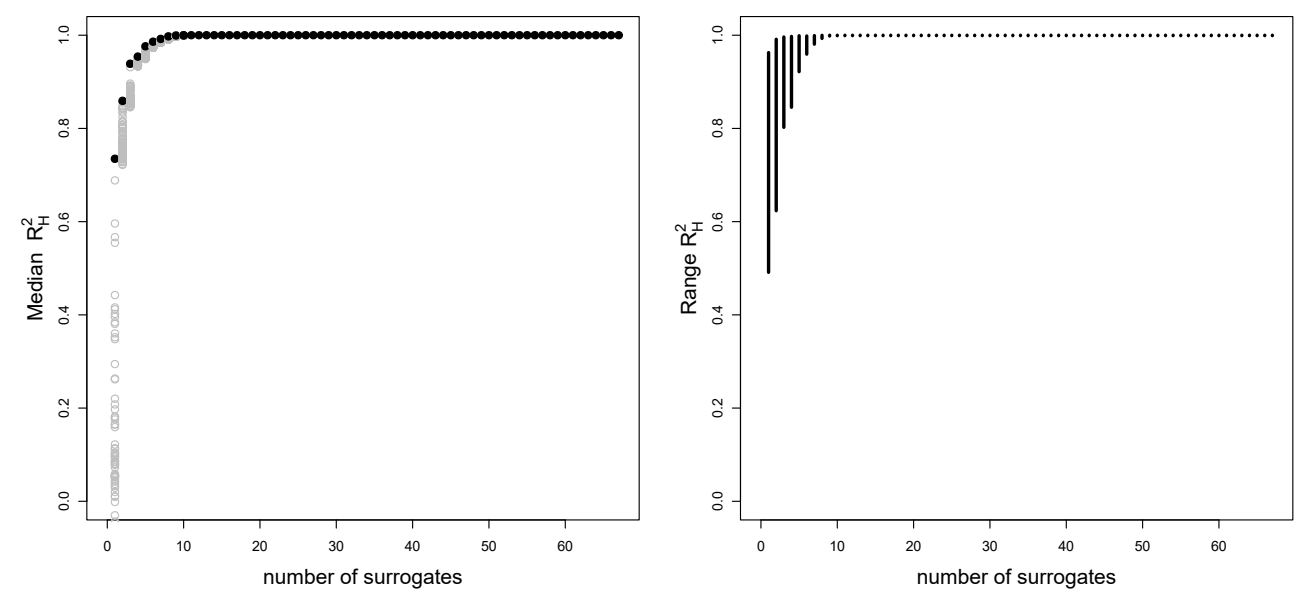

Fig. 2. TransPAT data. Median $R_{H}^{2}$ of all the sets of surrogates evaluated in each step of the forward selection (left hand side), where the black dot represents the highest median, and the grey dots the median of the rest of sets. $\left(\min R_{H}^{2}\right.$, $\max R_{H}^{2}$ ) interval of the best set of surrogate in each step (right hand side).

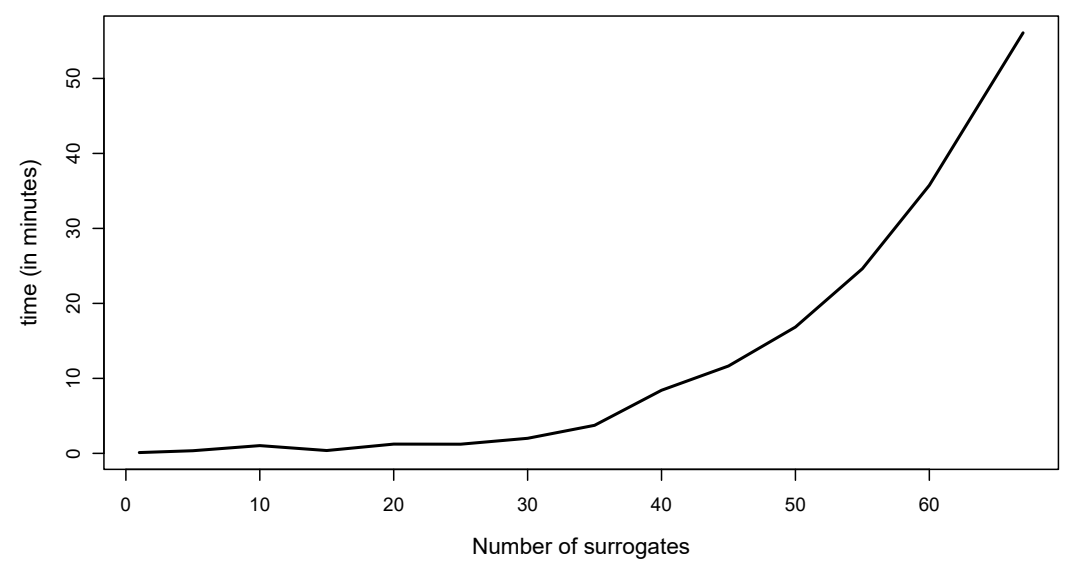

Fig. 3. TransPAT data. Median computation time to calculate $R_{H}^{2}$ based on 500 randomly generated correlation matrices. 
Table 1. Mean computation time (in seconds) to draw a correlation matrix with fixed values using different algorithms for various number of surrogates $(p)$ and fixed $\rho$.

\begin{tabular}{|c|c|c|c|c|c|c|c|c|c|c|c|c|}
\hline \multirow[b]{2}{*}{$p$} & \multicolumn{4}{|c|}{$\rho=0.2$} & \multicolumn{4}{|c|}{$\rho=0.5$} & \multicolumn{4}{|c|}{$\rho=0.8$} \\
\hline & GRS & $\mathrm{PC}$ & SHR & $\mathrm{SCA}$ & GRS & $\mathrm{PC}$ & SHR & SCA & GRS & $\mathrm{PC}$ & SHR & $\mathrm{SCA}$ \\
\hline$\overline{1}$ & 0.004 & 0.001 & 0.004 & 0.013 & 0.004 & 0.001 & 0.005 & 0.014 & 0.004 & 0.001 & 0.005 & $\overline{0.026}$ \\
\hline 3 & 0.011 & 0.004 & 0.019 & 0.18 & 0.117 & 0.003 & 0.024 & 0.207 & 0.512 & 0.003 & 0.052 & 0.199 \\
\hline 5 & 2.196 & 0.012 & 0.083 & 0.56 & 4.288 & 0.018 & 0.074 & 0.487 & 384.846 & 0.008 & 0.064 & 0.513 \\
\hline 10 & $\infty$ & 0.023 & 0.283 & 1.592 & $\infty$ & 0.034 & 0.36 & 1.861 & $\infty$ & 0.06 & 0.267 & 1.704 \\
\hline
\end{tabular}

Table 2. transPAT data. Percentage of cases in which the true ICA (coverage) was included in the [min, $\max ]$ range of ICA values computed using the PC algorithm.

\begin{tabular}{ccccccccccc}
\hline & & \multicolumn{1}{c}{ Number of runs $(\mathrm{M})$} \\
\cline { 3 - 11 } True ICA & $N$ & 100 & 200 & 500 & 1,000 & 2,000 & 5,000 & 10,000 & 20,000 & 50,000 \\
\hline 0.75 & 100 & 0.54 & 0.58 & 0.68 & 0.75 & 0.79 & 0.83 & 0.84 & 0.85 & 0.88 \\
& 200 & 0.59 & 0.67 & 0.76 & 0.82 & 0.87 & 0.92 & 0.94 & 0.94 & 0.95 \\
& 500 & 0.61 & 0.73 & 0.87 & 0.92 & 0.96 & 0.98 & 0.99 & 0.99 & 1.00 \\
\hline 0.85 & 100 & 0.98 & 0.98 & 0.99 & 0.99 & 1.00 & 1.00 & 1.00 & 1.00 & 1.00 \\
& 200 & 1.00 & 1.00 & 1.00 & 1.00 & 1.00 & 1.00 & 1.00 & 1.00 & 1.00 \\
& 500 & 1.00 & 1.00 & 1.00 & 1.00 & 1.00 & 1.00 & 1.00 & 1.00 & 1.00 \\
\hline 0.95 & 100 & 0.79 & 0.87 & 0.93 & 0.96 & 0.97 & 0.99 & 0.99 & 1.00 & 1.00 \\
& 200 & 0.85 & 0.94 & 0.98 & 1.00 & 1.00 & 1.00 & 1.00 & 1.00 & 1.00 \\
& 500 & 0.89 & 0.97 & 1.00 & 1.00 & 1.00 & 1.00 & 1.00 & 1.00 & 1.00 \\
\hline
\end{tabular}

